\title{
Throughput Analysis of Multiple Access Relay Channel under Collision Model
}

\author{
Seyed Amir Hejazi and Ben Liang
}

\begin{abstract}
Despite much research on the throughput of relaying networks under idealized interference models, many practical wireless networks rely on physical-layer protocols that preclude the concurrent reception of multiple transmissions. In this work, we develop analytical frameworks for the uplink of a multi-source single-channel relay-aided wireless system where transmissions are scheduled to avoid collisions. We study amplify-and-forward and decode-and-forward strategies, in both time-sharing and network-coded variants, and provide mathematical models to investigate their achievable rate regions. Both general and optimal power allocations are considered. We also find the cut-set outer bounds for the rate regions. Moreover, we present a comparison between these methods with the simple time sharing scheme. Our numerical results reveal that optimizing power allocation favors the time sharing scheme significantly more than it does the relaying schemes, so that time sharing under some circumstances can provide higher maximum sum rates, even if the links to the relay have strong channel gains. The proposed analysis provides a means to quantitatively evaluate the efficacy of relaying under the collision model, leading to pragmatic design guidelines.
\end{abstract}

\section{INTRODUCTION}

The pioneering work of Cover and El Gamal [1] introduced the concept of relaying in a single-transmitter single-relay network. Even though the exact performance characterization of this simplest relay network remains an open problem, the capacity region under some special conditions are known. For example, [2] gives the capacity regions for degraded and reversely degraded relays, as well as an achievable rate region and an outer bound for the more general case, which are the best bounds known to-date. Furthermore, much research has been devoted to understanding the effect of relays on the performance of various systems with respect to different network metrics [3]-[9]. In particular, [3] introduced an abstract representation of a relay network, termed the multiple access relay channel (MARC), which has received much further study. In MARC, multiple sources communicate with a destination node with assistance from one relay, which may represent, for example, the uplink of a relay-aided cellular network. Figure 1 illustrates such a network with two sources.

All of the above studies are based on physical-layer models that allow different nodes to transmit simultaneously, while accounting for the interference at the receiver as a weighted sum of the transmissions. One particularly strong yet commonly made assumption is the full-duplex communication of relays, such that a relay can receive packets while forwarding packets in the same physical channel at the same time. To implement such a relay in practice would require multiple

This work was supported in part by NSERC Discovery and Strategic Grants (RGPIN 262018 and STPSC 356786).

The authors are with the Department of Electrical and Computer Engineering, University of Toronto, Canada. Emails: \{seyedamir, liang\}@comm.utoronto.ca.

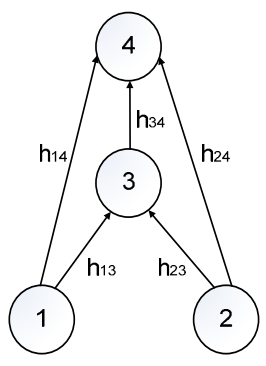

Fig. 1. Multiple access relay channel with two sources 1 and 2, relay 3, and destination 4.

antennas and possibly sophisticated coding and signal processing techniques. No widely deployed wireless technology, e.g., GSM, IEEE 802.11, or IEEE 802.16, supports this mode of operation. To fill this gap between theoretical models and practical networks, half-duplex relays, which cannot transmit and receive at the same time, have been analyzed in [7][9]. Sankaranarayanan et al. [7] derived outer bounds on the capacity region of a constrained MARC model, in which the relay operates in the receive and transmit state respectively for a fraction $\alpha$ and $1-\alpha$ of the time. In [8], Sankar et al. specialized these bounds to the orthogonal MARC channel with additive white Gaussian links, where the relay communicates in an orthogonal channel with the destination. They developed optimal power allocation policies that maximize the sum rate. Maric et al. [9] studied a two-sender, two-receiver channel model with one relay node. They derived the rate regions of several simple relaying strategies for a half-duplex relay that receives data in one block and transmits it to the destinations in the following block. In spite of the usage of half-duplex relays, these models still allow the relay and destination to receive data from multiple sources simultaneously.

Simultaneous packet reception is not impossible to achieve, but it generally requires sophisticated transmitters or receivers. For example, there are theoretical studies and practical implementations, reported in the literature, on multiuser detection through successive interference cancelation [10]-[12]. However, these techniques require that the received signals differ substantially either in power or in coding [13], [14]. It is also possible to perform distributed source beamforming [15], [16], where the sources form a virtual array of antennas to transmit simultaneously toward the destination, but this technique requires strict time synchronization among the sources at the symbol modulation level, and the same message must be transmitted by all sources. More recently, in [17], the authors proposed ZigZag decoding to allow the resolution of two colliding packets at the receiver in practical wireless networks with simple physical layer implementations. However, ZigZag decoding requires repeated transmissions whenever a collision 
occurs, so its applicability to relaying is unclear.

In this paper, we focus on relaying wireless networks with simple transceivers, where concurrent transmissions lead to packet collisions. We study the throughput capacity of MARC under the practical constraint where transmissions from different sources and the relay are scheduled to avoid collisions. This model in a single-source single-relay network was studied in [18]. Our work considers a wider variety of relaying strategies. Our main technical contributions are as follows:

- We develop analytical frameworks to study the achievable rate regions of amplify-and-forward (AF) and decodeand-forward (DF) in such an environment, with an AWGN channel model. For both cases, we consider timeshare forwarding, where the sources separately utilize the relay, and combined forwarding, where the relay forwards a linear combination of the source messages as a variant of network coding [19] in either the physical layer for $\mathrm{AF}$ or the network layer for DF.

- The time-sharing and network-coded AF and DF schemes provide inner bounds to the capacity region of this network. We further derive a cut-set outer bound (OB) for the capacity region and compare it with the above inner bounds. We demonstrate how the gap between these bounds vary given different channel conditions.

- We study the effect of power allocation in the above transmission strategies. Optimal power allocation to maximize the sum rate is considered for each strategy, and comparison is made with equal power allocation.

- We compare the rate regions of AF and DF with that of simple time sharing (TS), where the sources take turns to transmit directly to the destination without the relay. We show that optimizing power allocation favors TS significantly more than it does the relaying schemes. Our numerical results elucidate the channel conditions under which relaying is beneficial.

Different relaying strategies and power allocation schemes require different levels of implementation complexity. Hence, the proposed analytical frameworks and the subsequent numerical results provide a first step toward quantitative design guidelines on how to efficiently utilize relaying for throughput improvement. To the best of our knowledge, this work is the first analytical study on multiple access relaying networks under the collision model.

The rest of this paper is organized as follows. Section II describes our network model. In Section III, we discuss the transmission and relaying methods and derive the corresponding rate regions. The derivation of optimal power allocation, followed by a brief discussion on the results, will be presented in Section IV. In Section V, we provide numerical results under equal and optimal power allocation schemes with a detailed discussion on the performance of each strategy. Finally, we conclude the paper in Section VI.

\section{SySTEM MODEL}

We consider the MARC model as shown in Figure 1 and impose the constraint that only one node can transmit at a time in a broadcast manner. In this paper, we focus on the case of two source nodes transmitting to a destination in the presence of a relay. The proposed analysis in Sections III and IV can be extended to the general case with more than two sources, but that would involve tedious matrix manipulations and higherdimensional plots of rate regions that may add little technical insight.

We assume a common block coding scheme for message transmission [1], in which the nodes send their information in blocks of equal lengths. The links are assumed to experience additive white Gaussian noise, so that when node $i$ transmits the $n^{\text {th }}$ block $X_{i}^{(n)}$, node $j$ receives $Y_{j}^{(n)}$ given by

$$
Y_{j}^{(n)}=h_{i j} X_{i}^{(n)}+Z_{i j}^{(n)} \text {. }
$$

where $h_{i j}{ }^{1}$ is the channel gain from node $i$ to node $j$ and $Z_{i j}^{(n)}$ is an independent Gaussian random variable. We normalize all signal powers in the system, so that $Z_{i j}^{(n)}$ has unit variance. We denote by $P_{i}$ the upper limit on the average transmission power of node $i$, which can be chosen arbitrarily in general and will be optimized in Section IV. Then, the achievable rate, in bits per channel use, on link $i j$ is limited by the Shannon bound:

$$
R_{i j} \leq C\left(h_{i j}^{2} P_{i}\right) \triangleq \frac{1}{2} \log _{2}\left(1+h_{i j}^{2} P_{i}\right)
$$

The relay, when active, transmits $X_{3}^{(n)}$, based on previously received signals [1]:

$$
X_{3}^{(n)}=f\left(Y_{3}^{(n-1)}, \ldots, Y_{3}^{(1)}\right) .
$$

This encoding function can capture numerous relaying strategies, amongst which we will consider only AF and DF.

\section{Communication Strategies And Rate Regions}

In this section, we describe various communication strategies and present analysis to derive the achievable rate region for each. We denote the achievable rates of the two sources as $R_{1}$ and $R_{2}$, respectively.

\section{A. Time Sharing}

Without using the relay, the sources can time share the channel to transmit directly to the destination. Suppose the portions of time dedicated to source 1 and source 2 are $\alpha$ and $1-\alpha$ respectively. The rate region of TS is simply given by

$$
\begin{aligned}
R_{1} & \leq \alpha C\left(h_{14}^{2} P_{1}\right) \\
R_{2} & \leq(1-\alpha) C\left(h_{24}^{2} P_{2}\right) \\
0 & \leq \alpha \leq 1
\end{aligned}
$$

For the purpose of illustration in the rest of this paper, we are mainly interested in the case $\alpha=\frac{1}{2}$, where the sources share equal time.

\footnotetext{
${ }^{1}$ The channel gains, in general, are complex valued numbers; however, We are interested in their magnitude only. Therefore, throughout this paper $h_{i j}$ represents the magnitude of the channel gain.
} 


\section{B. Amplify-and-Forward}

Considering the constraint that only one node can transmit at a time, the relay has two options to assist the sources. It can either forward the two received blocks from the sources in separate time intervals, which we term Time-Sharing AF (TSAF), or it can merge the received blocks and transmit the combined signal, which we term Combined AF (CAF).

Time-Sharing AF: The network essentially operates as two sperate single-source, single-relay AF channels. We split the transmission time into four equal parts. Each source takes onefourth of the time to broadcast its block to the relay and the destination. The relay then takes one-fourth of the time to forward each of the two blocks.

Without loss of generality, suppose the sources transmit their blocks in every other one-fourth time interval. Whenever source $i$ transmits $X_{i}^{(n)}$ in one block, the relay receives $Y_{3}^{n}=h_{i 3} X_{i}^{(n)}+Z_{i 3}^{(n)}$. It then transmits $X_{3}^{(n+1)}=\alpha_{i} Y_{3}^{(n)}$ in the following block, where $\alpha_{i}$ is chosen to satisfy the relay's power constraint

$$
\alpha_{i} \leq \sqrt{\frac{P_{3}}{h_{i 3}^{2} P_{i}+1}} .
$$

Thus, the received signal at the destination in four consecutive blocks is

$$
\begin{aligned}
\mathrm{Y}_{4}^{(\mathrm{n})} & =\left[\begin{array}{c}
h_{14} \\
0 \\
\alpha_{1} h_{13} h_{34} \\
0
\end{array}\right] X_{1}^{(n-3)}+\left[\begin{array}{c}
0 \\
h_{24} \\
0 \\
\alpha_{2} h_{23} h_{34}
\end{array}\right] X_{2}^{(n-1)} \\
+ & {\left[\begin{array}{c}
Z_{14} \\
Z_{24} \\
\alpha_{1} h_{34} Z_{13}+Z_{34}^{(n-2)} \\
\alpha_{2} h_{34} Z_{23}+Z_{34}^{(n)}
\end{array}\right] . }
\end{aligned}
$$

Note that here and for the rest of this paper, we keep the block index of $Z_{i j}^{(n)}$ only when it is necessary to indicate the independence between noise in different blocks, and omit it whenever there is no risk of confusion.

The above equation describes a multiple access channel (MAC) with two transmitters and one receiver. Consequently, the capacity region satisfies [14]

$$
\begin{aligned}
R_{1} & \leq \frac{1}{4} C\left(\left(h_{14}^{2}+\frac{\alpha_{1}^{2} h_{13}^{2} h_{34}^{2}}{\alpha_{1}^{2} h_{34}^{2}+1}\right) P_{1}\right) \\
R_{2} & \leq \frac{1}{4} C\left(\left(h_{24}^{2}+\frac{\alpha_{2}^{2} h_{23}^{2} h_{34}^{2}}{\alpha_{2}^{2} h_{34}^{2}+1}\right) P_{2}\right) .
\end{aligned}
$$

The one-fourth factors above are the natural consequence of time sharing. Note that this capacity region defines a square rate region as opposed to a pentagon, which is the known rate region for the general MAC. The pentagon region of the general MAC is due to the sum-rate constraint as a direct consequence of the simultaneous reception of both inputs at the destination, which is not allowed here.

Combined AF: This strategy can be considered as analog network coding [19]. We split the transmission time into three equal parts. The sources each takes one-third of the time to broadcast their blocks, $X_{1}^{(n-1)}$ and $X_{2}^{(n)}$. Then, the relay transmits a linear combination of the received signals in the subsequent block

$$
X_{3}^{(n)}=\beta_{1} Y_{3}^{(n-2)}+\beta_{2} Y_{3}^{(n-1)},
$$

such that $\beta_{1}$ and $\beta_{2}$ satisfy the relay power constraint

$$
\beta_{1}^{2} P_{1}+\beta_{2}^{2} P_{2} \leq P_{3} \text {. }
$$

Consequently, the channel output at the destination in three successive blocks, for a given choice of the $\left(\beta_{1}, \beta_{2}\right)$ pair , is

$$
\begin{aligned}
\mathrm{Y}_{4}^{(\mathrm{n})} & =\left[\begin{array}{c}
h_{14} \\
0 \\
\beta_{1} h_{13} h_{34}
\end{array}\right] X_{1}^{(n-2)}+\left[\begin{array}{c}
0 \\
h_{24} \\
\beta_{2} h_{23} h_{34}
\end{array}\right] X_{2}^{(n-1)} \\
& +\left[\begin{array}{c}
Z_{14} \\
Z_{24} \\
\beta_{1} h_{34} Z_{13}+\beta_{2} h_{34} Z_{23}+Z_{34}
\end{array}\right]
\end{aligned}
$$

We can show that the resulting rate region is (see Appendix A)

$$
\begin{aligned}
R_{1} & \leq \frac{1}{3} C\left(\left(h_{14}^{2}+\frac{\beta_{1}^{2} h_{13}^{2} h_{34}^{2}}{\left(\beta_{1}^{2}+\beta_{2}^{2}\right) h_{34}^{2}+1}\right) P_{1}\right) \\
R_{2} & \leq \frac{1}{3} C\left(\left(h_{24}^{2}+\frac{\beta_{2}^{2} h_{23}^{2} h_{34}^{2}}{\left(\beta_{1}^{2}+\beta_{2}^{2}\right) h_{34}^{2}+1}\right) P_{2}\right) \\
R_{1} & +R_{2} \leq \frac{1}{3} C\left(\left(h_{14}^{2}+\frac{\beta_{1}^{2} h_{13}^{2} h_{34}^{2}}{\left(\beta_{1}^{2}+\beta_{2}^{2}\right) h_{34}^{2}+1}\right) P_{1}\right. \\
& +\left(h_{24}^{2}+\frac{\beta_{2}^{2} h_{23}^{2} h_{34}^{2}}{\left(\beta_{1}^{2}+\beta_{2}^{2}\right) h_{34}^{2}+1}\right) P_{2}+\left(h_{14}^{2} h_{24}^{2}\right. \\
& \left.\left.+\frac{\beta_{1}^{2} h_{13}^{2} h_{24}^{2} h_{34}^{2}}{\left(\beta_{1}^{2}+\beta_{2}^{2}\right) h_{34}^{2}+1}+\frac{\beta_{2}^{2} h_{23}^{2} h_{14}^{2} h_{34}^{2}}{\left(\beta_{1}^{2}+\beta_{2}^{2}\right) h_{34}^{2}+1}\right) P_{1} P_{2}\right)
\end{aligned}
$$

The overall rate region is the union of (9) over all possible choices of $\left(\beta_{1}, \beta_{2}\right)$ that satisfy (7). It is apparent that this rate region is a pentagon. In this regard, the combination of source messages at the relay has a similar effect as the simultaneous message reception in the general MAC.

\section{Decode-and-Forward}

Similar to the AF case, we consider two options for DF, termed Time-Sharing DF (TSDF) and Combined DF (CDF).

Time-Sharing DF: Similarly to TSAF, each source takes one-fourth of the time to broadcast its block to the relay and the destination. When source $i$ transmits $X_{i}^{(n)}$, the relay attempts to decode it and forward $a_{i} X_{i}^{(n)}$ in the next block, where $a_{i}$ satisfies

$$
a_{i} \leq \sqrt{\frac{P_{3}}{P_{i}}} .
$$

If relay decoding is always successful, the destination receives in four sequential blocks

$$
\mathrm{Y}_{4}^{(\mathrm{n})}=\left[\begin{array}{c}
h_{14} \\
0 \\
a_{1} h_{34} \\
0
\end{array}\right] X_{1}^{(n-3)}+\left[\begin{array}{c}
0 \\
h_{24} \\
0 \\
a_{2} h_{34}
\end{array}\right] X_{2}^{(n-1)}+\left[\begin{array}{c}
Z_{14} \\
Z_{24} \\
Z_{34}^{(n)} \\
Z_{34}^{(n-2)}
\end{array}\right]
$$

Again, the above equation describes a MAC with rate region [14]

$$
\begin{aligned}
R_{1} & \leq \frac{1}{4} C\left(\left(h_{14}^{2}+a_{1}^{2} h_{34}^{2}\right) P_{1}\right) \\
R_{2} & \leq \frac{1}{4} C\left(\left(h_{24}^{2}+a_{2}^{2} h_{34}^{2}\right) P_{2}\right) .
\end{aligned}
$$


To account for relay decoding failures, we note that the sources are independent and the transmissions occur separately. Therefore, the following rate constraints are imposed to the sourcerelay links separately:

$$
\begin{aligned}
R_{1} & \leq \frac{1}{4} C\left(\left(h_{13}^{2} P_{1}\right)\right. \\
R_{2} & \leq \frac{1}{4} C\left(\left(h_{23}^{2} P_{2}\right) .\right.
\end{aligned}
$$

Hence, the overall rate region is

$$
\begin{aligned}
& R_{1} \leq \frac{1}{4} \min \left\{C\left(\left(h_{13}^{2} P_{1}\right), C\left(\left(h_{14}^{2}+a_{1}^{2} h_{34}^{2}\right) P_{1}\right)\right\}\right. \\
& R_{2} \leq \frac{1}{4} \min \left\{C\left(\left(h_{23}^{2} P_{2}\right), C\left(\left(h_{24}^{2}+a_{2}^{2} h_{34}^{2}\right) P_{2}\right)\right\} .\right.
\end{aligned}
$$

Combined DF: This is a variant of the linear network coding approach [19]. As in CAF, we partition the transmission time into three equal portions among the sources and the relay. The sources each takes one-third of the time to broadcast their blocks, $X_{1}^{(n-1)}$ and $X_{2}^{(n)}$. Then, if and only if both blocks are decoded successfully, the relay transmits a linear combination of the blocks in the subsequent block

$$
X_{3}^{(n)}=b_{1} X_{1}^{(n-2)}+b_{2} X_{2}^{(n-1)},
$$

where $b_{1}$ and $b_{2}$ are chosen to satisfy the relay power constraint

$$
b_{1}^{2} P_{1}+b_{2}^{2} P_{2} \leq P_{3} .
$$

Hence, the destination receives in three sequential blocks

$$
Y_{4}^{(\mathrm{n})}=\left[\begin{array}{c}
h_{14} \\
0 \\
b_{1} h_{34}
\end{array}\right] X_{1}^{(n-2)}+\left[\begin{array}{c}
0 \\
h_{24} \\
b_{2} h_{34}
\end{array}\right] X_{2}^{(n-1)}+\left[\begin{array}{c}
Z_{14} \\
Z_{24} \\
Z_{34}
\end{array}\right]
$$

The rate region corresponding to (16) can be derived similarly to that of CAF. Further considering the relay decoding conditions in (13), the rate region of CDF for a specific choice of $\left(b_{1}, b_{2}\right)$ can be written as

$$
\begin{aligned}
R_{1} & \leq \frac{1}{3} \min \left\{C\left(h_{13}^{2} P_{1}\right), C\left(\left(h_{14}^{2}+b_{1}^{2} h_{34}^{2}\right) P_{1}\right)\right\} \\
R_{2} & \leq \frac{1}{3} \min \left\{C\left(h_{23}^{2} P_{2}\right), C\left(\left(h_{24}^{2}+b_{2}^{2} h_{34}^{2}\right) P_{2}\right)\right\} \\
R_{1} & +R_{2} \leq \frac{1}{3} \min \left\{C\left(h_{13}^{2} P_{1}\right)+C\left(h_{23}^{2} P_{2}\right),\right. \\
& C\left(\left(h_{14}^{2}+b_{1}^{2} h_{34}^{2}\right) P_{1}+\left(h_{24}^{2}+b_{2}^{2} h_{34}^{2}\right) P_{2}\right. \\
& \left.\left.+\left(h_{14}^{2} h_{24}^{2}+b_{1}^{2} h_{24}^{2} h_{34}^{2}+b_{2}^{2} h_{14}^{2} h_{34}^{2}\right) P_{1} P_{2}\right)\right\} .
\end{aligned}
$$

The overall rate region is the union of (17) over all choices of $\left(b_{1}, b_{2}\right)$ that satisfy (15). Similar to the CAF case, this rate region generally is also a pentagon.

\section{Outer Bound}

For comparison with the constructive inner bounds of the capacity region achieved by the communication strategies above, we develop a cut-set OB using the Max-Flow MinCut theorem [20]. It is clear from Figure 1 that two important cuts are the one that isolates the destination and the one that isolates the sources. Let $\gamma_{i}$ be the portion of time dedicated to node $i$, for $i=1,2,3$. The sum-capacity of these cuts leads to the following cut-set bound:

$$
\begin{gathered}
R_{1} \leq \gamma_{1} C\left(h_{14}^{2} P_{1}\right)+\min \left\{\gamma_{3} C\left(h_{34}^{2} P_{3}\right),\right. \\
\left.\gamma_{1} C\left(h_{13}^{2} P_{1}\right)+\gamma_{2} C\left(h_{23}^{2} P_{2}\right)\right\} \\
R_{2} \leq \gamma_{2} C\left(h_{24}^{2} P_{2}\right)+\min \left\{\gamma_{3} C\left(h_{34}^{2} P_{3}\right),\right. \\
\left.\left.\gamma_{1} C\left(h 13^{2} P_{1}\right)+\gamma_{2} C\left(h_{23}^{2} P_{2}\right)\right)\right\} \\
R_{1}+R_{2} \leq \gamma_{1} C\left(h_{14}^{2} P_{1}\right)+\gamma_{2} C\left(h_{24}^{2} P_{2}\right)+ \\
\min \left\{\gamma_{3} C\left(h_{34}^{2} P_{3}\right), \gamma_{1} C\left(h_{13}^{2} P_{1}\right)+\gamma_{2} C\left(h_{23}^{2} P_{2}\right)\right\} .
\end{gathered}
$$

The overall OB is the union of (18) over all choices of $\gamma_{i}$ that satisfy

$$
\begin{array}{r}
\gamma_{i} \geq 0, \quad i=1,2,3 \\
\sum_{i=1}^{3} \gamma_{i}=1 .
\end{array}
$$

Since equal-time block coding is used in AF and DF, we are also interested in the equal time-share outer bound (EOB) where $\gamma_{i}=\frac{1}{3}$ for all $i$. Section $\mathrm{V}$ provides a comparison between $\mathrm{OB}$ and $\mathrm{E}-\mathrm{OB}$.

\section{Optimal Power Allocation}

The analysis in the previous section assumes that $P_{i}$ for each source and relay node is given. Since wireless devices often have limited energy supply, in this section, we develop an optimization framework for allocating a limited sum of transmission power among the nodes, in order to maximize the achievable sum rate $R_{1}+R_{2}$. Suppose $P$ units of total power is available, the general form of our optimization problem is

$$
\begin{array}{cl}
\text { Maximize } & C_{R_{1}+R_{2}} \\
\text { Subject to } & P_{1}+P_{2}+P_{3} \leq P \\
& P_{i} \geq 0, i=1,2,3 \\
& g\left(P_{1}, P_{2}, P_{3}\right) \leq 0
\end{array}
$$

where $C_{R_{1}+R_{2}}$ is the sum-rate capacity, defined as the maximum achievable sum rate, given in Section III by the righthand side of the inequalities that describe the rate regions for different communication strategies, and $g\left(P_{1}, P_{2}, P_{3}\right) \leq 0$ represents the relay power constraint, expressed in (4), (7),(10), and (15) for TSAF, CAF, TSDF, and CDF, respectively. Note that for all relaying strategies, $g\left(P_{1}, P_{2}, P_{3}\right)$ is a linear function in $P_{i}$.Furthermore, it is easy to show that the sum-rate constraint in (9) and (17) dominates the individual rate constraints. Although the general formulation above is comparable to the well-known water-filling problem, the additional constraint on $\mathrm{g}($.$) prevent us from applying those results to our case.$

For TS, TSAF, and CAF, the sum-rate capacity consists of logarithmic functions. In these cases, the above optimization problem is convex. By applying Lagrangian multipliers and considering the Karush-Kuhn-Tucker conditions, we obtain the following optimal power allocations. The detailed derivations are provided in Appendix B. For TS, we have

$$
\begin{aligned}
& P_{1}=\left[\frac{P+h_{24}^{-2}-h_{14}^{-2}}{2}\right]^{+} \\
& P_{2}=\left[\frac{P+h_{14}^{-2}-h_{24}^{-2}}{2}\right]^{+} .
\end{aligned}
$$


For TSAF, we have

$$
\begin{aligned}
& P_{1}=\left[\frac{\left(\alpha_{2}^{2}-\alpha_{1}^{2}\right)\left(\alpha_{2}^{2} \frac{h_{23}^{2}}{2}+1\right)+\left(P-\frac{\alpha_{1}^{2}+\alpha_{2}^{2}}{2}\right) \alpha_{2}^{2} h_{23}^{2}}{\alpha_{1}^{2} h_{13}^{2}+\alpha_{2}^{2} h_{23}^{2}+\alpha_{1}^{2} \alpha_{2}^{2} h_{13}^{2} h_{23}^{2}}\right]^{+} \\
& P_{2}=\left[\frac{\left(\alpha_{1}^{2}-\alpha_{2}^{2}\right)\left(\alpha_{1}^{2} \frac{h_{13}^{2}}{2}+1\right)+\left(P-\frac{\alpha_{1}^{2}+\alpha_{2}^{2}}{2}\right) \alpha_{1}^{2} h_{13}^{2}}{\alpha_{1}^{2} h_{13}^{2}+\alpha_{2}^{2} h_{23}^{2}+\alpha_{1}^{2} \alpha_{2}^{2} h_{13}^{2} h_{23}^{2}}\right]^{+} \\
& P_{3}=\alpha_{1}^{2} h_{13}^{2} P_{1}+\alpha_{1}^{2},
\end{aligned}
$$

For CAF, we have

$$
\begin{aligned}
P_{1}= & {\left[\frac{-\frac{\beta_{2}^{2} h_{23}^{2}+1}{\beta_{1}^{2} h_{13}^{2}+1}\left(h_{14}^{2}+\frac{\beta_{1}^{2} h_{13}^{2} h_{34}^{2}}{\left(\beta_{1}^{2}+\beta_{2}^{2}\right) h_{34}^{2}+1}\right)+\left(h_{24}^{2}+\frac{\beta_{2}^{2} h_{23}^{2} h_{34}^{2}}{\left(\beta_{1}^{2}+\beta_{2}^{2}\right) h_{34}^{2}+1}\right)}{2\left(h_{14}^{2} h_{24}^{2}+\frac{\beta_{2}^{2} h_{14}^{2} h_{34}^{2} h_{23}^{2}+\beta_{1}^{2} h_{13}^{2} h_{24}^{2} h_{34}^{2}}{\left(\beta_{1}^{2}+\beta_{2}^{2}\right) h_{34}^{2}+1}\right)}\right.} \\
& \left.+\frac{P-\beta_{1}^{2}-\beta_{2}^{2}}{2\left(\beta_{1}^{2} h_{13}^{2}+1\right)}\right]^{+} \\
P_{2}= & {\left[\frac{\left(h_{14}^{2}+\frac{\beta_{1}^{2} h_{13}^{2} h_{34}^{2}}{\left(\beta_{1}^{2}+\beta_{2}^{2}\right) h_{34}^{2}+1}\right)-\frac{\beta_{1}^{2} h_{13}^{2}+1}{\beta_{2}^{2} h_{23}^{2}+1}\left(h_{24}^{2}+\frac{\beta_{2}^{2} h_{23}^{2} h_{34}^{2}}{\left(\beta_{1}^{2}+\beta_{2}^{2}\right) h_{34}^{2}+1}\right)}{2\left(h_{14}^{2} h_{24}^{2}+\frac{\beta_{2}^{2} h_{14}^{2} h_{34}^{2} h_{23}^{2}+\beta_{1}^{2} h_{13}^{2} h_{24}^{2} h_{34}^{2}}{\left(\beta_{1}^{2}+\beta_{2}^{2}\right) h_{34}^{2}+1}\right)}\right.} \\
& \left.\quad+\frac{P-\beta_{1}^{2}-\beta_{2}^{2}}{2\left(\beta_{2}^{2} h_{23}^{2}+1\right)}\right]^{+} \\
P_{3}= & \beta_{1}^{2} h_{13}^{2} P_{1}+\beta_{2}^{2} h_{23}^{2} P_{2}+\beta_{1}^{2}+\beta_{2}^{2}
\end{aligned}
$$

where $[\cdot]^{+}$denotes $\max \{0, \cdot\}$, ensuring that only non-negative power assignments are allowed. Note that the above solutions for TSAF and CAF are functions of the amplification parameters $\alpha_{1}, \alpha_{2}, \beta_{1}$, and $\beta_{2}$. Hence, to obtain the maximum sum rate, an additional step of numerical optimization over these parameters are necessary.

For TSDF and CDF, the minimum functions in the sumrate capacity leave us with a non-convex problem. In order to simplify the analysis in these cases, we consider only the condition that the channel gain of the links connecting the sources to the relay are sufficiently high, such that the overall sum rate is dominated (limited) by the sum rate from the sources and the relay to the destination. In this case, we can rewrite (14) as

$$
\begin{aligned}
& R_{1} \leq \frac{1}{4} C\left(\left(h_{14}^{2}+a_{1}^{2} h_{34}^{2}\right) P_{1}\right) \\
& R_{2} \leq \frac{1}{4} C\left(\left(h_{24}^{2}+a_{2}^{2} h_{34}^{2}\right) P_{2}\right) .
\end{aligned}
$$

and (17) as

$$
\begin{aligned}
R_{1} & \leq \frac{1}{3} C\left(\left(h_{14}^{2}+b_{1}^{2} h_{34}^{2}\right) P_{1}\right) \\
R_{2} & \leq \frac{1}{3} C\left(\left(h_{24}^{2}+b_{2}^{2} h_{34}^{2}\right) P_{2}\right) \\
R_{1} & +R_{2} \leq \frac{1}{3} C\left(\left(h_{14}^{2}+b_{1}^{2} h_{34}^{2}\right) P_{1}+\left(h_{24}^{2}+b_{2}^{2} h_{34}^{2}\right) P_{2}\right. \\
& \left.+\left(h_{14}^{2} h_{24}^{2}+b_{1}^{2} h_{24}^{2} h_{34}^{2}+b_{2}^{2} h_{14}^{2} h_{34}^{2}\right) P_{1} P_{2}\right) .
\end{aligned}
$$

This way, the power optimization problem for TSDF and CDF can be approximated by a convex problem. It is intuitive that, in general, the relay is beneficial only if it has a strong link to the sources. Therefore, the imposed condition still allows our analysis to be applicable to most of the important scenarios for TSDF and CDF. This is also confirmed in our numerical results in Section V.

Solving the convex versions of optimal power allocation in TSDF and CDF using a procedure similar to those for TSAF and $\mathrm{CAF}$, we have for TSDF

$$
\begin{aligned}
& P_{1}=\frac{P a_{1}^{-2}}{a_{1}^{-2}+a_{2}^{-2}+1} \\
& P_{2}=\frac{P a_{2}^{-2}}{a_{1}^{-2}+a_{2}^{-2}+1} \\
& P_{3}=\frac{P}{a_{1}^{-2}+a_{2}^{-2}+1},
\end{aligned}
$$

and for $\mathrm{CDF}$

$$
\begin{aligned}
& P_{1}=\left[\frac{P}{2\left(b_{1}^{2}+1\right)}+\frac{-\frac{b_{2}^{2}+1}{b_{1}^{2}+1}\left(h_{14}^{2}+b_{1}^{2} h_{34}^{2}\right)+\left(h_{24}^{2}+b_{2}^{2} h_{34}^{2}\right)}{2\left(h_{14}^{2} h_{24}^{2}+b_{1}^{2} h_{24}^{2} h 34^{2}+b_{2}^{2} h_{14}^{2} h_{34}^{2}\right)}\right]^{+} \\
& P_{2}=\left[\frac{P}{2\left(b_{2}^{2}+1\right)}+\frac{\left(h_{14}^{2}+b_{1}^{2} h_{34}^{2}\right)-\frac{b_{2}^{2}+1}{b_{1}^{2}+1}\left(h_{24}^{2}+b_{2}^{2} h_{34}^{2}\right)}{2\left(h_{14}^{2} h_{24}^{2}+b_{1}^{2} h_{24}^{2} h 34^{2}+b_{2}^{2} h_{14}^{2} h_{34}^{2}\right)}\right]^{+} \\
& P_{3}=b_{1}^{2} P_{1}+b_{2}^{2} P_{2} .
\end{aligned}
$$

We briefly discuss some implications of the above power optimization results in the following.

First, in all of the above relaying strategies, the optimal power allocation attempts to equalize the received SNR of each source at the destination. For example, in TSDF and CDF, we observe that the assigned powers are approximately proportional to the inverse of the dedicated power coefficients at the relay. Similar arguments can be made about TSAF and CAF. Hence, the optimal power policies, dispite the constraint on $g($.$) , have a water-filling [14] flavor in them.$

Second, unlike in relaying, the optimal power allocation in TS provides greater power for the channel with better conditions. In particular, the following relation exists:

$$
P_{1}-P_{2}=h_{24}^{-2}-h_{14}^{-2}
$$

so that $h_{14}>h_{24} \Rightarrow P_{1}>P_{2}$. This can be considered a form of opportunistic transmission [14].

Finally, equal power assignment in some scenarios is the optimal policy. e.g. In CDF, if we choose $b_{1}=b_{2}=\frac{1}{\sqrt{2}}$ and consider a symmetric model, where $h_{13}=h_{23}$, and $h_{14}=$ $h_{24}$, equation (27) results in equal assignment of powers, $P_{i}=$ $\frac{1}{3}, i=1,2,3$. We further study the performance of equal power allocation in Section $\mathrm{V}$.

\section{NumERICAL RESUlts}

We compute the rate regions and maximum sum rates, and present numerical results to illustrate the performance of different communication schemes. In all cases, we consider attenuating channels, where the channel gains are less than unity, since they are more likely to occur in practice than amplifying channels; however, the results in most cases can be extended to amplifying channels as well. In the following sub-sections, we first study the case of assigning power equally to all transmitters, then present the optimal power allocation results, and finally illustrate an example scenario where the sources and destination are fixed while the relay is moved along a line between them. 


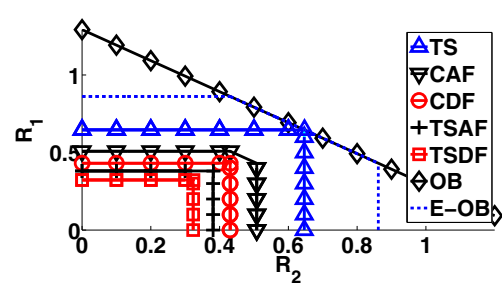

(a)

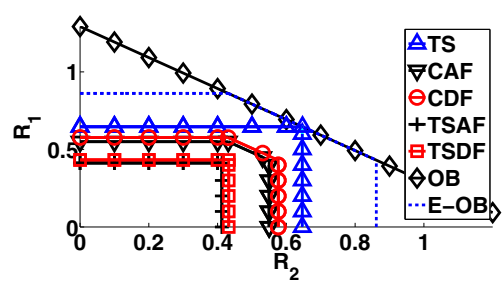

(d)

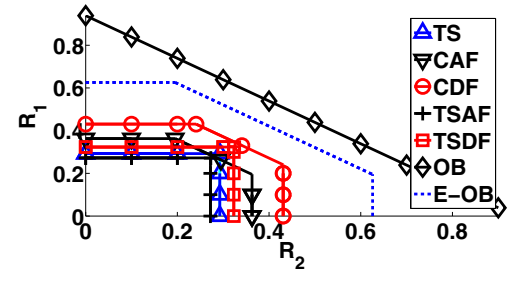

(b)

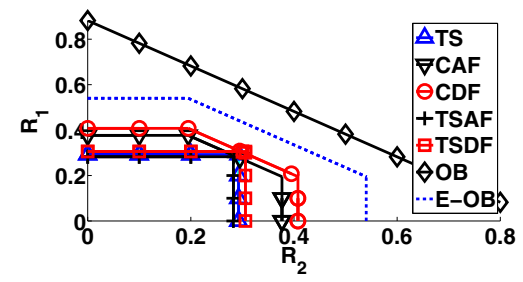

(e)

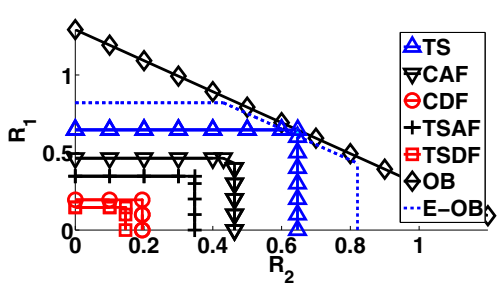

(c)

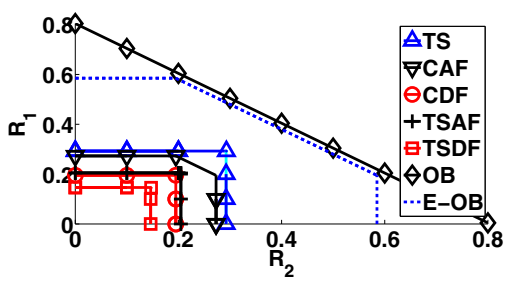

(f)

Fig. 2. Equal power allocation: impact of various channel conditions on the performance of each strategy. (a) $h_{14}=h_{24}=h_{13}=h_{23}=h_{34}=0.5$. (b) $h_{14}=h_{24}=0.25$ and $h_{13}=h_{23}=h_{34}=0.5$. (c) $h_{14}=h_{24}=h_{34}=0.5$ and $h_{13}=h_{23}=0.25$. (d) $h_{14}=h_{24}=h_{34}=0.25$ and $h_{13}=h_{23}=$ 0.5. (e) $h_{14}=h_{24}=0.25, h_{13}=h_{23}=0.9$, and $h_{34}=0.4$. (f) $h_{14}=h_{24}=h_{13}=h_{23}=0.25$ and $h_{34}=0.5$.

\section{A. Equal Power Allocation}

We assign equal powers, $P_{i}=20, i=1,2,3$, to each of the nodes $^{2}$. Figure 2 depicts the resulting rate regions under each communication strategy for different configuration of channel gains between the sources, relay, and destination. Figure 2(a): the channels have equal channel gains; 2(b): the direct links have the worst channels and all the links connected to the relay have equal channel gains; 2(c): the links between the sources and the relay have the worst conditions amongst all; 2(d): the links between the sources and the relay have better channel gains than others; 2(e): the relaying links have better conditions than the direct links, and the links between the sources and the relay do not limit the rate; and 2(f): the channel gain of the link between the relay and the destination is dominant.

We can observe from Figures 2(a) and (c) that the TS rate region far exceeds the rate regions of the relaying strategies. This is an intuitive result, since in these scenarios the channel gains of the direct link is strong compared with the relay links. Furthermore, we observe that the TS rate region touches the outer bound at $R_{1}=R_{2}$, indicating that TS is in fact the optimal communication strategy when the direct links are strong.

In Figures 2(b) and (e), the links that join the relay to the other nodes have better conditions than the direct links from the sources to the destination. It is apparent that in these cases the relay strategies give better performance than TS. Moreover, CDF operates close to the E-OB, which suggests that, under the requirement of service fairness (i.e., equal time sharing between sources), there may be no pragmatic need for more elaborate relaying schemes than CDF. CDF dominates all other communication strategies, using only a simple network coding approach. This exemplifies the efficacy of networklayer relaying compared to the physical-layer approaches such as TSAF and CAF. Furthermore, CAF and CDF in these

\footnotetext{
${ }^{2}$ Recall that all powers are normalized to have the unit of one noise variance.
}

cases provide much better rate regions than TSAF and TSDF respectively. This suggests the benefit of network coding if relaying is used.

Figures 2(d) and (f) illustrate the circumstances where either the links that connect the sources to the relay or the link that joins the relay to the destination have better states than the other ones. We see TS still has the dominant rate region in these cases. In spite of the relay having similar channel gains as direct links, all relaying strategies use a smaller portion of the total transmission time to send fresh information to the destination, which leads to degradation in the performance. Therefore, we conclude that, under the collision model, the relay is beneficial only when both incoming and outgoing links at the relay are strong.

\section{B. Optimal Power Allocation}

Next, we study the effect of optimal power allocation. We set the total available power to $P=60$. For fair comparison between the various communication strategies, we choose channel gains the same as the scenario of Figure 2(e), where the relay links have better channel conditions than the direct links. We distribute the available power optimally among the relay and sources as explained in Section IV.

Figure 3 demonstrates the achievable rate regions of different communication strategies, under power allocation schemes that maximizes the sum rate. For TS, equal time sharing is assumed by default. For the relaying strategies, we plot the rate regions for different $\alpha_{i}, \beta_{i}, a_{i}$, and $b_{i}$ values. An important observation here is that, under optimal powers, there is a drastic expansion of the TS rate region from the case of equal power allocation. In contrast, CDF, the best relaying strategy under equal power allocation, experiences little rate improvement.

Another interesting observation is the dominance of TSDF over other relaying methods in term of the maximum sum rate. The rate region of TSDF is larger than the rate regions of other 


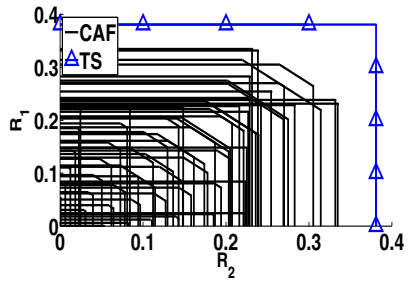

(a)

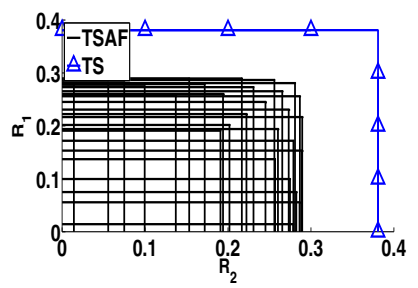

(c)

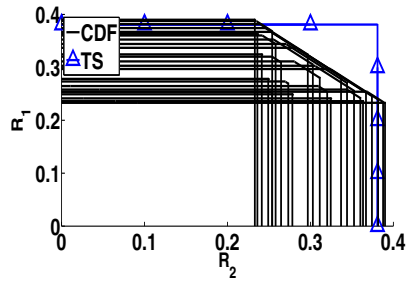

(b)

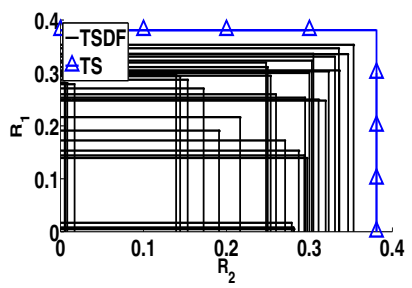

(d)
Fig. 3. Optimal power allocation: The shaded regions represent the acheivable rate regions under each relaying strategy, and the boundary of the TS' rate region is specified by the cyan color line.

strategies except CDF, but TSDF reaches far better sum rates than CDF. Note that the overall rate regions in Figure 3 may not necessarily be larger than those in Figure 2(e), because of the constraint of sum-rate maximization.

Furthermore, on the one hand, the fact that the sources have less time to transmit in TSDF compared with CDF and yet TSDF can achieve better sum-rate, stresses the importance of power allocation. On the other hand, the fact that the sources have more time to transmit in CDF than in TSDF, and CDF achieves better individual rates for the sources, suggests the potential for joint power, time, and coding design. Overall, comparing Figure 2(e) with Figure 3, we can conclude that, with appropriate allocation of system resources, time and power in this case, one can reduce the need for relaying.

\section{Case Study: Moving Relay}

In this section, we present a case study, where the channel gains are determined by the distance from a moving relay to the sources and the destination. We compare the sum-rate performance of all communication strategies to select the best strategy given the location of the relay.

Consider the symmetric structure of Figure 1, where the sources and the destination form an isosceles triangle, and the relay can be placed anywhere on the perpendicular bisector that passes through the destination. Let $d_{1}$ represent half of the distance between the two sources, and $d_{2}$ represent the distance of the destination to each source. We define the position of the relay in terms of its distance to the midpoint of the line joining the sources, and compute the maximum sum-rate achievable by each strategy for any position of the relay.

We consider different channel gains given by transmission path loss:

$$
h_{i j}=d_{i j}^{-\frac{r}{2}}
$$

where $d_{i j}$ is the distance between the nodes $i$ and $j$, and $r$ is the path-loss exponent. Again, for equal power allocation,

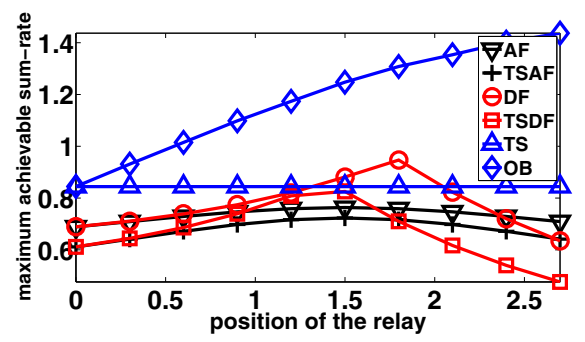

(a) Equal power, $r=2, d_{1}=0.1$ and $d_{2}=3$

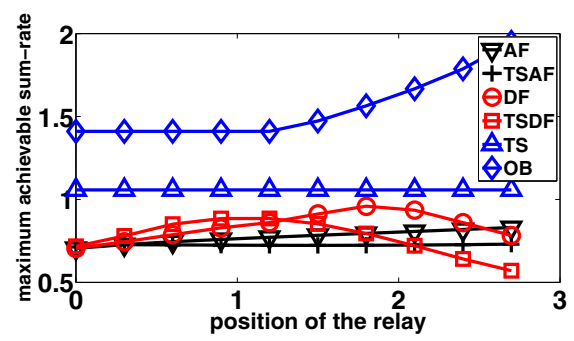

(b) Optimal power, $r=2, d_{1}=0.1$ and $d_{2}=3$

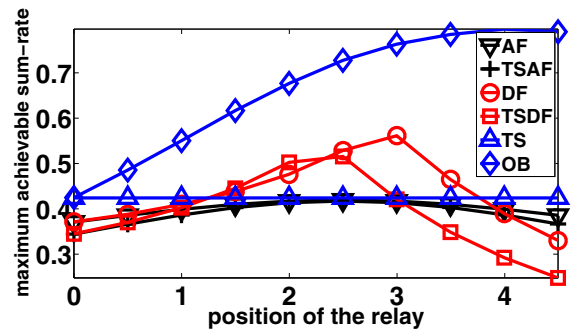

(c) Equal power, $r=2, d_{1}=0.25$ and $d_{2}=5$

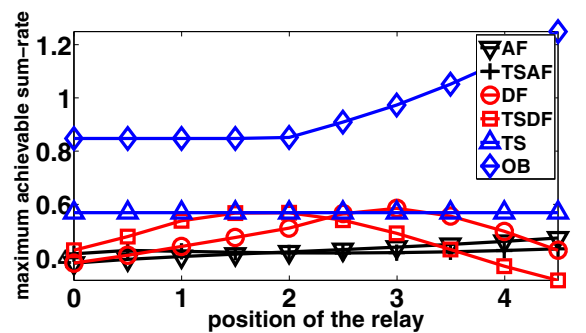

(d) Optimal power, $r=2, d_{1}=0.25$ and $d_{2}=5$

Fig. 4. Moving relay, with low path loss.

we assume $P_{1}=P_{2}=P_{3}=20$, and for optimal power allocation, we assume $P=60$.

Figures 4 and 5 demonstrate the numerical results under equal and optimal power allocations, for low path loss and high path loss, respectively. Note that parts of the CDF and TSDF curves for the optimal power case are cutoff in some plots, for relay positions that do not satisfy the channel conditions leading to (24) and (25).

Both figures show that, for all scenarios, whenever the relay is close to the sources or the destination, TS out performs the other strategies, even though the relay has one strong link either to the sources or to the destination. This observation validates the results obtained from Figures 2(d) and (f). For the relay to be beneficial, both incoming and outgoing links must be stronger than the direct source-destination link. In contrast, these figures show that the relay strategies, especially CDF and TSDF, can be helpful in the intermediate positions, when 


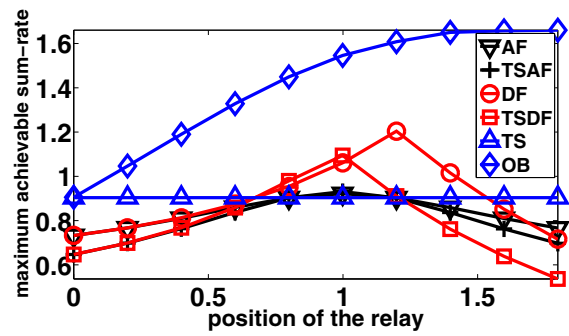

(a) Equal power, $r=3, d_{1}=0.1$ and $d_{2}=2$

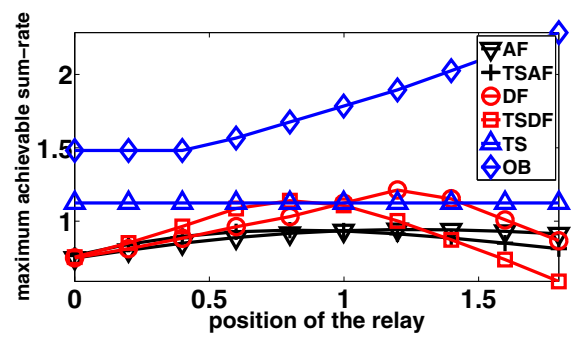

(b) Optimal power, $r=3, d_{1}=0.1$ and $d_{2}=2$

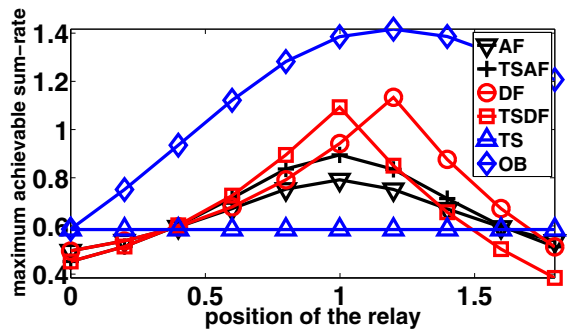

(c) Equal power, $r=4, d_{1}=0.1$ and $d_{2}=2$

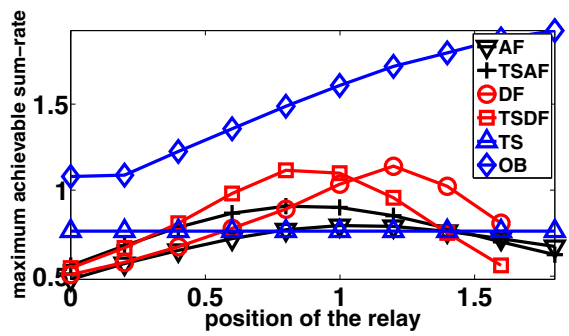

(d) Optimal power, $r=4, d_{1}=0.1$ and $d_{2}=2$

Fig. 5. Moving relay: with high path loss.

the above condition is satisfied.

Another interesting observation is the improvement to TS sum rates under power optimization, such that it can dominate all relaying strategies in some cases, no matter where the relay is, as shown in Figure 4(b). Optimal power allocation reduces the advantage of relaying, even when the relay is in the middle between the sources and the destination, so that the channel gains for the relay is strong. In other words, TS exploits better scheduling and power management to alleviate the impact of lower channel gains. Note that, although the relaying strategies that we have investigated are not the only possible ones, the proximity of the TS rates to the outer bound in some cases, and the overhead cost of more elaborate relaying strategies, may still obviate the need for relaying.

As we increase the channel path loss exponent, Figure 5 suggests that even the optimal allocation of resources is not sufficient to support TS. Under both, equal or optimal allocation of power, the DF strategies show better performance over the AF strategies and TS. Furthermore, the relative performance between TSDF and CDF depends on the location of the relay. TSDF uses a smaller amount of time to transmit fresh information to the destination in comparison with CDF; however, it can provide stronger copies of the transmitted data for the destination by dedicating more power to the individual messages of the sources. The performance advantage of TSDF for some relay positions suggests the trade off between time and power in different relaying strategies.

Finally, we observe that CDF always has the highest zenith point amongst all relaying strategies, so that if the relay location is free to choose by the system designer, $\mathrm{CDF}$ is the overall best relaying strategy. This, again, reflects the benefit of network coding based schemes. The proposed analysis additionally provides quantitative design guidelines on where to optimally place the relay for different relaying and coding strategies.

\section{CONCLUSION}

In this paper, we study a multiple access relay network under the collision model where concurrent transmissions are not allowed. We present various relaying strategies, including time-sharing $\mathrm{AF}$ and $\mathrm{DF}$, and variants of linear network coding in the physical layer and the network layer respectively combined with AF and DF. We develop their achievable rate regions and compare them with that of source time sharing without the relay and with cut-set outer bounds of the capacity region. We further derive optimal power allocation policies for each communication strategy. Our numerical results demonstrate that optimal power control policies under some conditions can obviate the need for relaying, which emphasizes the importance of resource management in the networks under consideration. Comparing the rate regions under equal and optimal power allocations gives us insights on the amount of performance gain that optimal power control policies can introduce, in comparison with the amount of complexity that they add to our network.

\section{APPENDIX A PROOF OF CAF RATE REgION}

Equation (8) describes a multiple access channel (MAC) with two transmitters and one receiver. Hence, the rate pair $\left(R_{1}, R_{2}\right)$ should satisfy

$$
\begin{aligned}
R_{1} & \leq \frac{1}{3} \max _{p\left(x_{1}\right) p\left(x_{2}\right)} I\left(X_{1} ; Y \mid X_{2}\right) \\
R_{2} & \leq \frac{1}{3} \max _{p\left(x_{1}\right) p\left(x_{2}\right)} I\left(X_{2} ; Y \mid X_{1}\right) \\
R_{1} & +R_{2} \leq \frac{1}{3} \max _{p\left(x_{1}\right) p\left(x_{2}\right)} I\left(X_{1}, X_{2} ; Y\right)
\end{aligned}
$$

where $p\left(x_{i}\right)$ is the probability function of $X_{i}$, and $I(A ; B)=$ $H(A)-H(A \mid B)$ where $H($.$) is the relative entropy function.$ The one-third fraction in front of each inequality is the consequence of time sharing between the nodes. 
For the first inequality, we have

$$
\begin{aligned}
& I\left(X_{1} ; Y \mid X_{2}\right)=H\left(Y \mid X_{2}\right)-H\left(Y \mid X_{1}, X_{2}\right) \\
& \quad=H\left(\left[\begin{array}{c}
h_{14} \\
0 \\
\beta_{1} h_{13} h_{34}
\end{array}\right] X_{1}^{n-3}+\left[\begin{array}{c}
Z_{14} \\
Z_{24} \\
h_{34}\left(\beta_{1} Z_{13}+\beta_{2} Z_{23}\right)+Z_{34}
\end{array}\right]\right) \\
& \quad-H\left(\left[\begin{array}{c}
Z_{14} \\
Z_{24} \\
h_{34}\left(\beta_{1} Z_{13}+\beta_{2} Z_{23}\right)+Z_{34}
\end{array}\right]\right)
\end{aligned}
$$

Under the hypothesis of an AWGN channel model, $H\left(Y \mid X_{1}, X_{2}\right)=C\left((2 \pi e)^{3} \operatorname{det} K_{Z}-1\right)$, where $K$ represents the covariance matrix. Furthermore, we have $H\left(Y \mid X_{2}\right) \leq$ $C\left((2 \pi e)^{3} \operatorname{det} K_{X_{1}, Z}-1\right)$. Hence,

$I\left(X_{1} ; Y \mid X_{2}\right) \leq C\left((2 \pi e)^{3} \operatorname{det} K_{X_{1}, Z}-1\right)-C\left((2 \pi e)^{3} \operatorname{det} K_{Z}-1\right)$

$$
\begin{aligned}
& \operatorname{det}\left[\begin{array}{ccc}
h_{14}^{2} P_{1}+1 & 0 & \beta_{1} h_{13} h_{14} h_{34} P_{1} \\
0 & 1 & 0 \\
\beta_{1} h_{13} h_{14} h_{34} P_{1} & 0 & \beta_{1}^{2} h_{13}^{2} h_{34}^{2} P_{1}+h_{34}^{2}\left(\beta_{1}^{2}+\beta_{2}^{2}\right)+1
\end{array}\right] \\
\operatorname{det}\left[\begin{array}{ccc}
1 & 0 & 0 \\
0 & 1 & 0 \\
0 & 0 & h_{34}^{2}\left(\beta_{1}^{2}+\beta_{2}^{2}\right)+1
\end{array}\right] & \\
\Rightarrow & R_{1} \leq \frac{1}{3} C\left(\left(h_{14}^{2}+\frac{\beta_{1}^{2} h_{13}^{2} h_{34}^{2}}{h_{34}^{2}\left(\beta_{1}^{2}+\beta_{2}^{2}\right)+1}\right) P_{1}\right) .
\end{aligned}
$$

The other rate region boundaries can be derived similarly from the other inequalities. This completes the proof of the rate region (9).

\section{APPENDIX B}

\section{Derivation of Optimal Power Allocations}

We show the derivation of optimal power allocation for CAF. The derivation for TS, TSAF, and modified versions of TSDF and CDF are similar and are omitted due to page limitations.

Denote by $\mathbf{P}$ the vector of power allocations, and $\lambda$ the vector of Lagrangian multipliers. The Lagrangian function of our problem is

$$
\begin{aligned}
L(\mathbf{P}, \lambda) & =-C_{R_{1}+R_{2}}-\sum_{i=1}^{3} \lambda_{i} P_{i}+\lambda_{4}\left(P_{1}+P_{2}+P_{3}-P\right) \\
& +\lambda_{5}\left(\beta_{1}^{2} h_{13}^{2} P_{1}+\beta_{2}^{2} h_{23}^{2} P_{2}+\beta_{1}^{2}+\beta_{2}^{2}-P_{3}\right) .
\end{aligned}
$$

Note that the last term above corresponds to the relay power constraint specific to CAF, as shown in (7). To ensure $P_{i}>$ $0, i=1,2,3$, the complementary slackness imposes $\lambda_{i}=$ $0, i=1,2,3$. Applying the KKT conditions to (33) will result in,

$$
\begin{aligned}
\frac{\partial L}{\partial P_{3}} & =0 \Rightarrow \lambda_{4}=\lambda_{5} \\
\frac{\partial L}{\partial P_{1}} & =\frac{\partial L}{\partial P_{2}}=0 \overbrace{}^{\lambda_{4}=\lambda_{5}} \frac{1}{\beta_{2}^{2} h_{23}^{2}+1}\left(k p_{1}\right)-\frac{1}{\beta_{1}^{2} h_{13}^{2}+1}\left(k p_{2}\right) \\
= & \frac{1}{\beta_{1}^{2} h_{13}^{2}+1}\left(h_{14}^{2}+\frac{\beta_{1}^{2} h_{13}^{2} h_{34}^{2}}{h_{34}^{2}\left(\beta_{1}^{2}+\beta_{2}^{2}\right)+1}\right) \\
& -\frac{1}{\beta_{2}^{2} h_{23}^{2}+1}\left(h_{24}^{2}+\frac{\beta_{2}^{2} h_{23}^{2} h_{34}^{2}}{h_{34}^{2}\left(\beta_{1}^{2}+\beta_{2}^{2}\right)+1}\right)
\end{aligned}
$$

where $k=h_{14}^{2} h_{24}^{2}+\frac{\beta_{2}^{2} h_{14}^{2} h_{23}^{2} h_{34}^{2}}{h_{34}^{2}\left(\beta_{1}^{2}+\beta_{2}^{2}\right)+1}+\frac{\beta_{1}^{2} h_{24}^{2} h_{13}^{2} h_{34}^{2}}{h_{34}^{2}\left(\beta_{1}^{2}+\beta_{2}^{2}\right)+1}$. The choice of $\lambda_{4}=\lambda_{5}=0$ is not acceptable, since it imposes $P_{i}=$
$0, i=1,2,3$. Therefore, $\lambda_{i}>0$, for $i=4,5$, and from complementary slackness

$$
\begin{aligned}
P & =P_{1}+P_{2}+P_{3} \\
P_{3} & =\beta_{1}^{2} h_{13}^{2} P_{1}+\beta_{2}^{2} h_{23}^{2} P_{2}+\beta_{1}^{2}+\beta_{2}^{2}
\end{aligned}
$$

Equations (34) and (35) describe a system of linear equations. The solution of this system determines the optimal power allocation.

\section{REFERENCES}

[1] T. Cover and A. Gamal, "Capacity theorems for the relay channel," IEEE Transactions on Information Theory, vol. 25, no. 5, pp. 572-584, Sep 1979.

2] T. M. Cover and J. A. Thomas, Elements of Information Theory 2nd Edition (Wiley Series in Telecommunications and Signal Processing). Wiley-Interscience, July 2006.

[3] G. Kramer and A. van Wijngaarden, "On the white gaussian multipleaccess relay channel," in Proc. IEEE International Symposium on Information Theory (ISIT'00), 2000, pp. 40-.

[4] G. Kramer, M. Gastpar, and P. Gupta, "Cooperative strategies and capacity theorems for relay networks," IEEE Transactions on Information Theory, vol. 51, no. 9, pp. 3037-3063, Sept. 2005.

[5] M. Gastpar and M. Vetterli, "On the capacity of wireless networks: the relay case," in Proc. Twenty-First Annual Joint Conference of the IEEE Computer and Communications Societies INFOCOM'02, vol. 3, 2002, pp. 1577-1586 vol.3.

[6] J. Laneman, D. Tse, and G. Wornell, "Cooperative diversity in wireless networks: Efficient protocols and outage behavior," IEEE Transactions on Information Theory, vol. 50, no. 12, pp. 3062-3080, Dec. 2004.

[7] L. Sankaranarayanan, G. Kramer, and N. Mandayam, "Hierarchical sensor networks: capacity bounds and cooperative strategies using the multiple-access relay channel model," in Proc. First Annual IEEE Communications Society Conference on Sensor and Ad Hoc Communications and Networks (SECON'04), Oct. 2004, pp. 191-199.

[8] L. Sankar, Y. Liang, H. V. Poor, and N. Mandayam, "Opportunistic communications in an orthogonal multiaccess relay channel," in Proc. IEEE International Symposium on Information Theory (ISIT'07), June 2007, pp. 1261-1265.

[9] I. Maric, A. Goldsmith, and M. Medard, "Information-theoretic relaying for multicast in wireless networks," in Proc. IEEE Military Communications Conference (MILCOM'07), Oct. 2007, pp. 1-7.

[10] P. Castoldi, Multiuser Detection in CDMA Mobile Terminals. Norwood, MA, USA: Artech House, Inc., 2002.

[11] J. Andrews, "Interference cancellation for cellular systems: a contemporary overview," IEEE Wireless Communications, vol. 12, no. 2, pp. 19-29, April 2005.

[12] J. Hou, J. Smee, H. Pfister, and S. Tomasin, "Implementing interference cancellation to increase the ev-do rev a reverse link capacity," IEEE Communications Magazine, vol. 44, no. 2, pp. 58-64, Feb. 2006.

[13] S. Verdu, Multiuser Detection. New York, NY, USA: Cambridge University Press, 1998.

[14] D. Tse and P. Viswanath, Fundamentals of wireless communication. New York, NY, USA: Cambridge University Press, 2005.

[15] G. Barriac, R. Mudumbai, and U. Madhow, "Distributed beamforming for information transfer in sensor networks," in Proc. International Symposium on Information Processing in Sensor Networks (IPSN'04). New York, NY, USA: ACM, 2004, pp. 81-88.

[16] H. Ochiai, P. Mitran, H. Poor, and V. Tarokh, "Collaborative beamforming for distributed wireless ad hoc sensor networks," IEEE Transactions on Signal Processing, vol. 53, no. 11, pp. 4110-4124, Nov. 2005.

[17] S. Gollakota and D. Katabi, "ZigZag decoding: combating hidden terminals in wireless networks," in Proc. ACM SIGCOMM'08, 2008.

[18] R. Nabar, H. Bolcskei, and F. Kneubuhler, "Fading relay channels: performance limits and space-time signal design," IEEE Journal on Selected Areas in Communications, vol. 22, no. 6, pp. 1099-1109, Aug. 2004.

[19] R. Ahlswede, N. Cai, S.-Y. Li, and R. Yeung, "Network information flow," IEEE Transactions on Information Theory, vol. 46, no. 4, pp. 1204-1216, Jul. 2000.

[20] D. B. West, Introduction to Graph Theory (2nd Edition). Prentice Hall, August 2000. 